\title{
Comment on "Electron states for gapped pseudospin-1 fermions in the field of a charged impurity"
}

\author{
R. Van Pottelberge ( $^{*}$ \\ Departement Fysica, Universiteit Antwerpen, Groenenborgerlaan 171, B-2020 Antwerpen, Belgium
}

(Received 27 January 2020; revised manuscript received 4 March 2020; accepted 6 May 2020; published 19 May 2020)

\begin{abstract}
In a recent paper [Phys. Rev. B 99, 155124 (2019)], the spectrum of a regularized Coulomb charge was studied in gapped pseudospin-1 systems generated by an $\alpha-\mathcal{T}_{3}$ lattice. The electronic spectrum was studied as a function of the impurity strength $Z \alpha$. However, the results and conclusions on the behavior of the flatband states as a function of the impurity strength are incomplete. In this Comment, I argue that because of the dispersionless nature of the flatband, the states spread out under the influence of a charged impurity forming a continuous band of states. I support my arguments with explicit numerical calculations which show the emergence of a continuum of states.
\end{abstract}

DOI: 10.1103/PhysRevB.101.197102

In a recent paper [1], one of the main points of investigation was how a Coulomb potential affects flatband states. Especially, in the case of the Dice model $(\Theta=\pi / 4)$, they find that the flatband does not survive under a Coulomb impurity and that bound states split off and eventually sink in the negative continuum with increasing charge of the impurity. However, the authors presented an incomplete picture on the behavior of the flatband states under a regularized Coulomb impurity. In this Comment, I argue, based on both intuitive arguments and explicit finite element calculations, that the flatband states form a continuum as a function of the Coulomb charge. In this Comment, I limit myself to the most relevant case of the Dice model for which $\Theta=\pi / 4$ and which exhibits a band that is perfectly flat [see Fig. 1(a)].

As discussed in previous theoretical [2-5] works, the occurrence of flatband states leads to very peculiar physics. For a flatband, the kinetic energy is completely quenched (and the effective mass is infinite) with infinite degeneracy, and this can be, for example, seen as the divergence of the density of states (see Fig. 4 of Ref. [1]). Consequently, it is always possible to construct a linear combination of flatband states in such a way that the particle becomes infinitely localized [4,5]. In essence, the flatband consists of an infinite number of degenerate states which are perfectly localized at one specific point in space. These infinitely localized wave packets remain eigenstates of the single-particle Hamiltonian [5]. Thus, in the presence of a Coulomb charge, the flatband states will undergo a constant shift determined by the value of the Coulomb potential at the position of the particle. Consequently, the flatband states are expected to spread out as a continuum of states where the position of the lowest state is determined by the value of the Coulomb potential at the center of the charge. Whereas the top of the continuum is composed of states located infinitely far away from the Coulomb impurity that barely undergoes a shift. In Fig. 1(b), we show schematically how the spectrum will behave under the influence of a regularized

*robbe.vanpottelberge@uantwerpen.be charge $V(r)=-Z \alpha_{0} / \sqrt{r^{2}+r_{0}^{2}}$ as considered in Ref. [1] with $Z \alpha_{0}$ as the strength of the Coulomb potential and $r_{0}$ as the regularization parameter. The orange continuum allows for bound states exactly as in the case of gapped graphene (shown by the orange curves) [6]. However, the flatband (indicated in blue) spreads out and forms a continuum as a function of the impurity charge. The bottom of this band is given by $E=-Z \alpha_{0} / r_{0}$. The green band corresponds to hole states and, consequently, does not allow for bound states in the case of a positive impurity charge.

Our argument, based on the nature of the flatband states, also explains why the flatband survives in the case of the step potential well but not in the case of a Coulomb potential. In the case of a step potential, electronic states infinitely localized outside the well will not undergo a shift remaining at $E=$ 0 . In contrast, for the Coulomb potential, there is always a long-range tail which means that even particles infinitely far away from the charge will feel a, albeit small, shift effectively destroying the flatband at $E=0$.

So far I have only given intuitive arguments based on the nature of the flatband states. In order to confirm my arguments, I performed explicit numerical calculations. I solved the following set of dimensionless differential equations numerically using the finite elments method, see Eqs. (19) and (20) in Ref. [1]:

$$
\begin{gathered}
\left(-\frac{Z \alpha}{\sqrt{\rho^{2}+1^{2}}}+\bar{m}\right) \psi_{1}+\cos \Theta\left(\frac{\partial}{\partial \rho}+\frac{j}{\rho}\right) \psi_{2}=\epsilon \psi_{1}, \\
-\cos \Theta\left(\frac{\partial}{\partial \rho}-\frac{(j-1)}{\rho}\right) \psi_{1}-\frac{Z \alpha}{\sqrt{\rho^{2}+1^{2}}} \psi_{2} \\
-\sin \Theta\left(\frac{\partial}{\partial \rho}+\frac{(j+1)}{\rho}\right) \psi_{3}=\epsilon \psi_{2}, \\
\sin \Theta\left(\frac{\partial}{\partial \rho}-\frac{j}{\rho}\right) \psi_{2}+\left(-\frac{Z \alpha}{\sqrt{\rho^{2}+1^{2}}}-\bar{m}\right) \psi_{3}=\epsilon \psi_{3} .
\end{gathered}
$$

Here, $\rho=r / r_{0}, \epsilon=E r_{0} / \hbar v_{F}, \bar{m}=m r_{0} / \hbar v_{F}, \quad$ and $\alpha=$ $\alpha_{0} / \hbar v_{F}$. In my calculations, I took the Dice model for which 
(a)

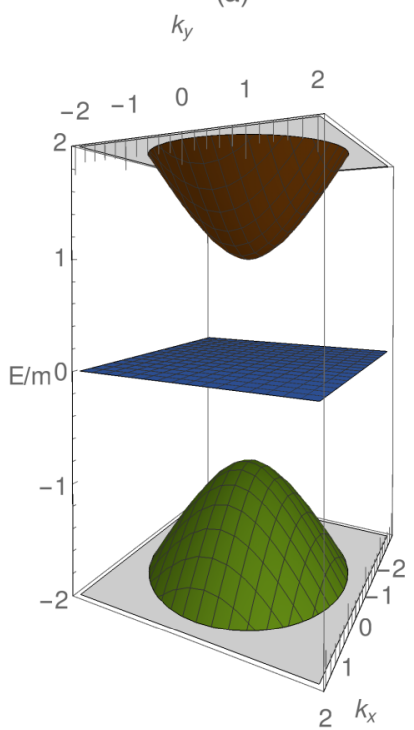

(b)

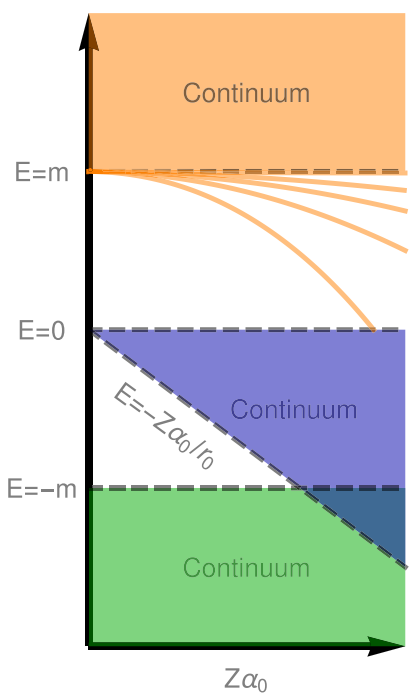

FIG. 1. (a) Zero field band structure of the gapped Dice model which exhibits a perfect flatband (blue) at $E=0$. (b) Schematic of how the bands will behave under the influence of a charged impurity.

$\Theta=\pi / 4$. For computational simplicity, I used a regularized Coulomb potential of the form $V(r)=-Z \alpha_{0} / \sqrt{r^{2}+r_{0}^{2}}$. Note that the exact form of the regularized potential does not matter.

My calculations were performed using the finite elements method implemented in Mathematica where the lattice is discretized and the coupled differential equations (1)-(3) are solved giving the eigenvalues and eigenfunctions. I solve the set of coupled equations on a descretized lattice from 0 to $600 r_{0}$. In Fig. 2, the results are plotted for two mesh sizes $\left(0.5 r_{0}\right.$ blue and $2 r_{0}$ red), $\bar{m}=0.08$ and $j=0$. I observe a number of states originating from $E=0$ as expected from

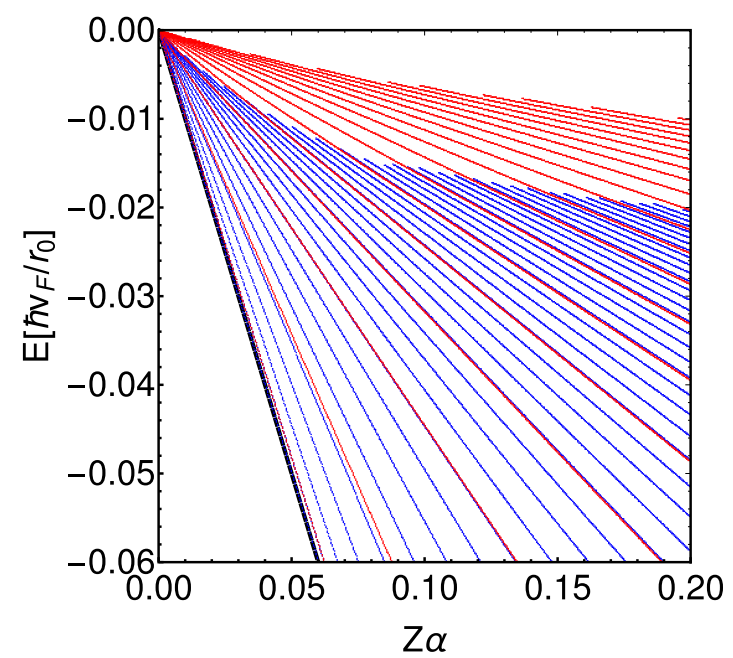

FIG. 2. Finite elements calculation on a lattice from 0 to $600 r_{0}$ for two different kinds of mesh sizes (blue $0.5 r_{0}$ and red $2 r_{0}$ ). For $\bar{m}$, we took the values of 0.08 and $j=0$. The black line denotes $E=-Z \alpha$.

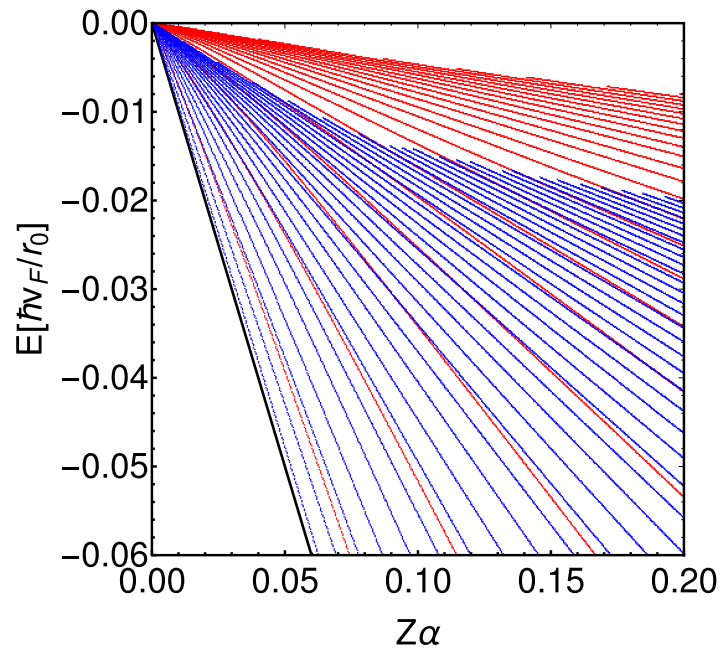

FIG. 3. The same as in Fig. 2 but for $j=2$.

my intuitive arguments. I observe that, with decreasing mesh size (from red to blue in Fig. 2), the density of states as a function of energy increases. This means that the finite elements method picks ups a continuum originating from $E=0$ (otherwise, it would converge to a fixed number of levels). I, indeed, observed that decreasing the mesh size even further leads to the emergence of even more states closer to each other than the blue ones observed in Fig. 2. Interestingly, none of states cross the line $E\left[\hbar v_{F} / r_{0}\right]=-Z \alpha$, indicated as the black line in Fig. 2. This line exactly corresponds to the argument made that the lowest state should behave as $E=$ $-Z \alpha_{0} / r_{0}\left(E=-Z \alpha\right.$ in units of $\left.\hbar v_{F} / r_{0}\right)$ made previously. I find a similar result for higher angular momentum states as shown in Fig. 3 for $j=2$. In short, the fact that the finite elements method picks up a continuum together with the fact that none of the numerically calculated states dive below $E=-Z \alpha$ confirms my intuitively made argument. Note that the gap between the top state and $E=0$ observed in Fig. 2 is a consequence from the fact that the eigenvalue solver is only able to find a finite number of solutions. I would also like to

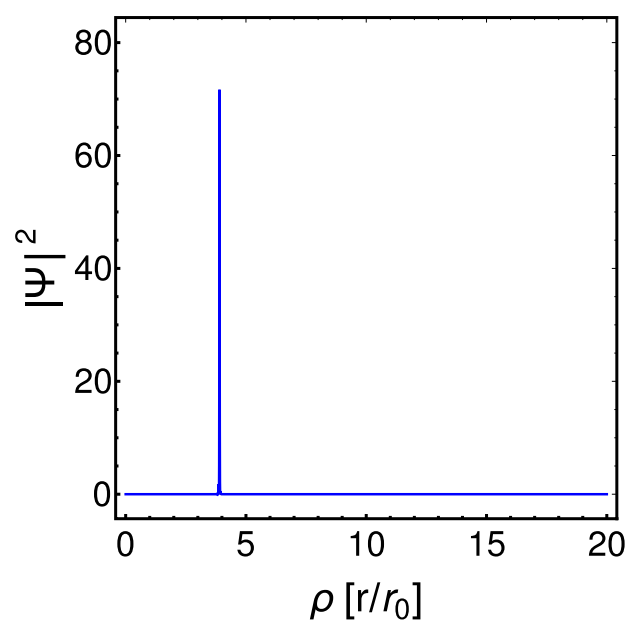

FIG. 4. Typical spatial profile of a state calculated in Fig. 2 shows a sharp localization. 
note that, although the numerical solutions approximate the real solutions, they technically do not represent real physical states for a finite mesh.

In Fig. 4, I show the spatial distribution obtained from the finite elements method for one of the states shown in Fig. 2. A very sharp and distinct peak can be observed confirming my intuitive arguments previously made. The value of the wave function increases with decreasing mesh size retaining its form as seen in Fig. 4.

In this Comment, I argued that the spectrum for a gapped Dice model under an impurity charge calculated in Ref. [1] is incomplete. I argued that, based on the dispersionless properties of the flatband and the states being completely localized, that no states split of but, instead, a continuum as a function of the charge $Z \alpha_{0}$ will be formed. I supported my arguments by explicit numerical finite elements calculations. I showed that the finite elements find a number of states originating from
$E=0$ which never cross $E=-Z \alpha_{0} / r_{0}$ and move closer to each other with decreasing mesh size. This behavior indicates the emergence of a continuum originating from the flatband as a function of the impurity strength. To conclude, I would like to make the remark that the Hamiltonian solved in this Comment constitutes a continuum limit of the Dice model and should not be confused with the real lattice of the Dice model. For future work, it would be interesting to study the problem in a tight-binding context which models the real Dice lattice.

I would like to acknowledge very insightful discussions with the authors of the commented paper (V. P. Gusynin, E. V. Gorbar, and D. O. Oriekhov). F. M. Peeters is acknowledged for interesting discussions and proofreading. This research was supported by the Flemish Science Foundation through an aspirant research grant for R.V.P.
[1] E. V. Gorbar, V. P. Gusynin, and D. O. Oriekhov, Phys. Rev. B 99, 155124 (2019).

[2] J. Sabio, F. Sols, and F. Guinea, Phys. Rev. B 81, 045428 (2010).

[3] A. Raoux, M. Morigi, J.-N. Fuchs, F. Piechon, and G. Montambaux, Phys. Rev. Lett. 112, 026402 (2014).
[4] M. Tovmasyan, S. Peotta, L. Liang, P. Törmä, and S. D. Huber, Phys. Rev. B 98, 134513 (2018).

[5] Doron L. Bergman, C. Wu, and L. Balents, Phys. Rev. B 78, 125104 (2008).

[6] R. Van Pottelberge, B. Van Duppen, and F. M. Peeters, Phys. Rev. B 98, 165420 (2018). 